COMUNICACIÓN CORTA

\title{
Factores asociados al uso, disposición y eliminación de elementos de protección personal y otros insumos de la clínica veterinaria de grandes animales
}

\author{
Signorini $\mathrm{ML}^{1,2}$, Molineri $\mathrm{Al}^{1,3,4}$, Meléndez Orantes $\mathrm{CE}^{5}$, Tarabla $\mathrm{HD}^{2 *}$ \\ ${ }^{1}$ Consejo Nacional de Investigaciones Científicas y Técnicas (CONICET) (Argentina). \\ ${ }^{2}$ Facultad de Ciencias Veterinarias, Universidad Nacional del Litoral (FCV-UNL) (Argentina). \\ ${ }^{3}$ Instituto Nacional de Tecnología Agropecuaria (INTA) EEA Rafaela, Santa Fe (Argentina). \\ ${ }^{4}$ Universidad Nacional de Rafaela (Argentina). \\ ${ }^{5}$ Facultad de Ciencias Veterinarias, Universidad Nacional de Rosario (Argentina).
}

\author{
* Correspondencia: FCV-UNL, R.P. Kreder 2805, Esperanza (3080), Santa Fe, \\ Argentina. \\ E-mail: htarabla@fcv.unl.edu.ar
}

Recibido: 30 Abril 2019. Aceptado: 6 Junio 2019. Disponible en línea: 20 Junio 2019

Editor: P. Beldomenico

RESUMEN. Se efectuó un estudio transversal en 330 Médicos Veterinarios de grandes animales en la Provincia de Santa Fe, Argentina para estimar: a) frecuencia de uso y disposición final de elementos de protección personal e insumos para la práctica clínica, b) asociaciones con características sociodemográficas de los entrevistados. El análisis de los datos incluyó: $\chi 2, t$ de Student, test exacto de Fisher y correlación de Pearson. El 30,8\% había sido diagnosticado con zoonosis (brucelosis $27,2 \%$ ). El descarte de los guantes a campo fue la forma de disposición más frecuente. Uno de cada cinco profesionales los eliminaba junto a la basura domiciliaria y cuatro de cada 10 hacía lo propio con las agujas descartables. El género y la edad de los profesionales estuvieron significativamente asociados con la utilización, disposición y eliminación de los insumos veterinarios luego de diversas prácticas profesionales.

\begin{abstract}
SUMMARY. Factors associated with the usage, disposal and elimination of personal protective equipment and other veterinary inputs in large animal practice. A cross-sectional study was carried out in 330 veterinarians working on large animal practice in the Province of Santa Fe, Argentina to estimate: a) frequency of usage and final disposal of personal protective equipment and supplies for clinical practice, $b$ ) associations with socio-demographic characteristics of the interviewees. Data analysis included: $\chi 2$, Student's $t$ test, Fisher's exact test and Pearson's correlation coefficient. Thirty percent had been diagnosed with zoonosis (brucellosis: 27.2\%). Discarding gloves on the farm was the most frequent means for disposal. One out of five practitioners eliminated the gloves along with household wastes, while four out of 10 had a similar behavior on regards of disposable needles. Practitioners' gender and time after graduation were significantly associated with usage, disposal and elimination of veterinary inputs following various professional practices.
\end{abstract}

Palabras clave: clínica de grandes animales, residuos veterinarios, disposición y eliminación

Keywords: large animal practice, veterinary wastes, disposal and elimination

Los veterinarios tienen un alto riesgo de contraer una zoonosis, servir inadvertidamente como centinelas biológicos de enfermedades emergentes y potencialmente propagar agentes patógenos (Baker y Gray, 2009). Sin embargo, la adopción de prácticas seguras en la práctica profesional es baja y la disposición final de los desechos generados es a menudo cuestionable (Tarabla,
2017). Si bien son generadores de residuos, los veterinarios pueden tener poca conciencia del problema e incumplir con la legislación vigente (McLean et al., 2007). En Argentina, aunque la Ley Nacional № 24.051 / 91 y la mayoría de las legislaciones provinciales consideran como desechos biopatogénicos los residuos clínicos de la atención médica, algunas provincias solo 
se refieren a establecimientos de salud humana y otros con características similares, sin ser explícitas en la inclusión de los centros de atención veterinaria (de Titto et al., 2015). Los objetivos de este estudio fueron: a) cuantificar la frecuencia de uso y disposición final de algunos elementos de protección personal (EPP) e insumos para la práctica clínica, b) buscar asociaciones con características sociodemográficas de los entrevistados.

Se efectuó un estudio transversal en Médicos Veterinarios de grandes animales en la Provincia de Santa Fe, Argentina. Un total de $n=330$ encuestados respondieron anónimamente un cuestionario estructurado (error absoluto del 4,7\%, nivel de confianza 95\%, frecuencia previa del $50 \%$, ajustado por el tamaño de la población $\mathrm{N}=1400$ estimado según los padrones de afiliados y de corresponsables sanitarios en los Colegios Veterinarios de Santa Fe $1^{\text {era }}$ y $2^{\text {da }}$ Circ.). Las variables estudiadas fueron: uso, disposición y descarte de EPP (guantes, overol y botas de goma en la vacunación antibrucélica, atención de partos, manipulación de abortos y necropsias) y otros insumos veterinarios (agujas hipodérmicas descartables y cuchillo de necropsia). Las características sociodemográficas de los entrevistados incluyeron: edad, género, antigüedad profesional, actividad laboral (sólo grandes animales, grandes y pequeños, grandes y otra) y zona de ejercicio laboral (Norte, Centro y Sur provincial). Para la búsqueda de asociaciones con el uso, disposición y eliminación de insumos veterinarios, la antigüedad profesional fue transformada en dicotómica tomando como punto de corte la mediana. El análisis de los datos incluyó: $\chi^{2}, t$ de Student, test exacto de Fisher $y$ correlación de Pearson.

Los encuestados tenían $43,9 \pm 10,9$ años de edad y $18,0 \pm 11,6$ años (mediana 17 años) de antigüedad profesional. Ambas variables estuvieron altamente correlacionadas $(R=0,97 ; P<0,001)$, por lo que en la búsqueda de asociaciones sólo se utilizó la antigüedad profesional. La gran mayoría $(96,4 \%)$ eran hombres, de mayor edad que las mujeres $(P=0,01)$. El $68,0 \%$ sólo trabajaba con grandes animales, $27,4 \%$ junto a clínica de pequeños animales y $4,6 \%$ junto a otras actividades profesionales. El 1,6\% había adquirido brucelosis cuando era estudiante de veterinaria. El 30,8\% había sido diagnosticado al menos con una zoonosis a lo largo de su trayectoria profesional, siendo brucelosis $(27,2 \%)$ la más frecuente. El 11,7\% había padecido más de una zoonosis. En general, el uso de EPP fue más frecuente en la manipulación de abortos y necropsias. El descarte de los guantes a campo fue la forma de disposición más frecuente, mientras que una minoría desinfectaba los overoles y las botas previo al lavado y luego de la actividad profesional, respectivamente (Tabla 1). La contaminación bacteriana puede sobrevivir en telas y contaminar superficies de todo tipo por contacto breve representando un peligro potencial importante. Además, algunos desinfectantes como los hipocloritos pueden no ser confiables cuando las telas están muy contaminadas (Scott, 1990).

La eliminación de los guantes junto a la basura domiciliaria fue practicada por, aproximadamente uno de cada cinco profesionales (Tabla 2). La disposición de estos desechos como residuos domésticos comprometen la salud de los recolectores de basura y de quienes remueven los desperdicios como una forma de subsistencia (McLean et al., 2007).

La reutilización o el uso inapropiado de elementos punzocortantes es una práctica frecuente en medicina de grandes animales (Huertas et al., 2018, Wright et al., 2008), donde las heridas por punción son muy frecuentes (Navarrete y Tarabla, 2018, López Cepeda et al., 2014, Signorini et al., 2014). Las agujas descartables fueron reutilizadas frecuentemente (siempre $8,1 \%$, a veces $53,5 \%$, nunca $38,4 \%$ ). El $38,0 \%$ las eliminaba con la basura domiciliaria, $17,8 \%$ las enterraba, $44 \%$ las quemaba y $15,0 \%$ acudía a otras prácticas. El $8,3 \%$ se valía de una práctica altamente desaconsejable como lo es la utilización del cuchillo de necropsias para otras actividades. Por otra parte, el 7,7\% lo esterilizaba y el $50,3 \%$ lo desinfectaba "siempre", mientras que el resto sólo lo hacía "a veces". Tanto en la disposición como en la eliminación de estos insumos, algunos profesionales utilizaban más de una forma de ejecutarlas. A modo de ejemplo, una proporción variable del total de encuestados que guardaban los guantes para disponer de ellos con posterioridad o nunca reusaban las agujas descartables, también descartaban los guantes a campo (vacunación antibrucélica 9,6\%; atención de partos $11,8 \%$; manipulación de abortos $10,0 \%$ y necropsias $10,9 \%)$ o incluían las agujas junto a la basura domiciliaria $(37,8 \%)$, respectivamente. Esto indica claramente la carencia, en estos casos, de un protocolo estándar para la disposición final de estos insumos. El uso de EPP in labore es más frecuente en mujeres y jóvenes profesionales (Navarrete y Tarabla, 2018, Tarabla et al., 2017). Coincidentemente, estos grupos usaron más frecuentemente los EPP y adoptaron más prácticas seguras de disposición y eliminación de los insumos de trabajo que los hombres y los de mayor antigüedad laboral, con la única excepción de la relación entre esta última variable y el descarte de guantes a campo (Tabla 3). No hubo diferencias significativas en la desinfección de las botas luego de su uso y el lavado por separado del overol entre profesionales de distinto género o antigüedad laboral. Tampoco se detectaron asociaciones con la actividad o la zona de ejercicio profesional. Los estudios observacionales no sirven para determinar causalidad, sino para generar nuevas hipótesis. El escaso número de profesionales del género femenino impidió estimar el nivel de significancia de algunas asociaciones y efectuar un análisis multivariado para dilucidar si las mujeres son más prudentes en su trabajo profesional per se, o es una observación relacionada sólo con la menor edad y antigüedad profesional en relación a los hombres. Estas últimas variables fueron un importante factor de confusión en la relación 
entre género y la frecuencia de brucelosis en los veterinarios rurales de la región (Molineri et al., 2013), hipótesis que será probada en nuestras investigaciones actuales.

Los veterinarios pueden rechazar el uso de barreras protectoras por su incomodidad, la falta de disponibilidad, los costos adicionales, o la creencia que tienen un bajo riesgo de enfermar con una zoonosis (Baker y Gray, 2009). La seguridad laboral de estos profesionales exige mejor equipamiento, condiciones $y$ rutinas de trabajo. Por otra parte, la falta de adhesión a prácticas seguras de disposición y eliminación de los insumos utilizados puede estar asociada a la ignorancia de la legislación existente (McLean et al., 2007), la falta de acceso a empresas autorizadas para el descarte de residuos peligrosos (Imoberdorf et al., 2017), o al desconocimiento y falta de observación de normas mínimas de prevención (Tarabla et al., 2017). En estas instancias, se requieren mejores programas de educación continua para minimizar los riesgos ocupacionales y ambientales, haciendo hincapié en los estudiantes de veterinaria y los jóvenes profesionales.

Tabla 1. Disposición de elementos de protección personal luego de diversas prácticas profesionales, Santa Fe, Argentina ( $\mathrm{n}=330$ ). Las sumas no totalizan el $\mathbf{1 0 0} \%$ porque los encuestados adoptaron diversas combinaciones de utilización y disposición de los insumos en cada actividad.

\begin{tabular}{|c|c|c|c|c|c|}
\hline \multirow[b]{2}{*}{ Insumo } & \multirow[b]{2}{*}{ Utilización/ disposición } & \multicolumn{4}{|c|}{ Actividad } \\
\hline & & $\begin{array}{l}\text { Vacunación } \\
\text { antibrucélica }\end{array}$ & Atención de partos & Manejo de abortos & Necropsias \\
\hline \multirow[t]{3}{*}{ Guantes } & Descarta a campo & 56,7 & 66,0 & 61,2 & 62,2 \\
\hline & Guarda y descarta & 44,8 & 35,1 & 41,7 & 37,7 \\
\hline & Reutiliza & 9,1 & 7,5 & 6,0 & 2,8 \\
\hline Overol & Desinfecta & 6,4 & 7,9 & 8,7 & 7,9 \\
\hline \multirow[t]{2}{*}{ Botas } & Usa siempre & 52,7 & 77,6 & 75,3 & 78,6 \\
\hline & Desinfecta & 13,1 & 14,5 & 15,2 & 16,9 \\
\hline
\end{tabular}

Tabla 2. Eliminación de los guantes luego de diversas prácticas profesionales, Santa Fe, Argentina ( $n=330)$. Las sumas no totalizan el $100 \%$ porque los encuestados adoptaron diversas combinaciones de utilización y disposición de los insumos en cada actividad.

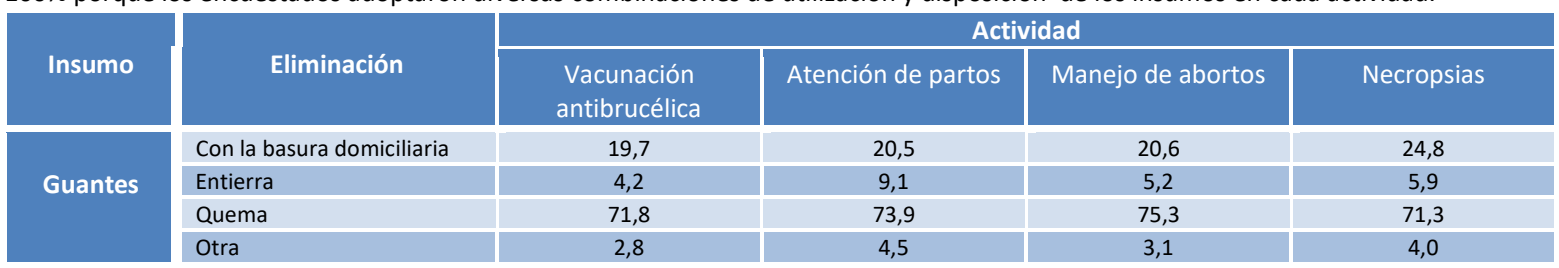

Tabla 3. Utilización, disposición y eliminación de insumos luego de diversas prácticas profesionales, Santa Fe, Argentina ( $n=330$ ).

\begin{tabular}{|c|c|c|c|c|c|c|c|c|}
\hline \multirow{3}{*}{ Insumo } & \multirow{3}{*}{ Actividad } & \multirow{3}{*}{$\begin{array}{c}\text { Utilización/ disposición/ } \\
\text { eliminación }\end{array}$} & \multicolumn{6}{|c|}{ Variables independientes (\% de encuestados) } \\
\hline & & & \multicolumn{3}{|c|}{ Años de graduado } & \multicolumn{3}{|c|}{ Género } \\
\hline & & & $\leq 17$ & $>17$ & $P$ & Hombre & Mujer & $P$ \\
\hline \multirow{12}{*}{ Guantes } & \multirow{3}{*}{$\begin{array}{l}\text { Vacunación contra } \\
\text { brucelosis }\end{array}$} & Usa siempre & 52,1 & 35,1 & 0,002 & 42,7 & 75,0 & 0,027 \\
\hline & & Descarta a campo & 62,5 & 48,9 & 0,049 & 59,3 & 9,1 & 0,001 \\
\hline & & Basura domiciliaria & 12,5 & 34,8 & 0,052 & 21,2 & 0 & - \\
\hline & \multirow[t]{3}{*}{ Atención de partos } & Usa siempre & 81,8 & 58,1 & 0,001 & 69,7 & 91,7 & 0,192 \\
\hline & & Descarta a campo & 69,8 & 61,4 & 0,130 & 67,6 & 25,0 & 0,004 \\
\hline & & Basura domiciliaria & 13,5 & 30,6 & 0,051 & 21,7 & 0 & - \\
\hline & \multirow[t]{3}{*}{ Manejo de abortos } & Usa siempre & 95,1 & 88.5 & 0,030 & 91,9 & 91,7 & 0,981 \\
\hline & & Descarta a campo & 66,7 & 42,0 & 0,040 & 62,6 & 20,0 & 0,016 \\
\hline & & Basura domiciliaria & 11,3 & 31,8 & 0,013 & 21,7 & 0 & - \\
\hline & \multirow{3}{*}{ Necropsia } & Usa siempre & 94,5 & 87,4 & 0,027 & 90,9 & 100,0 & - \\
\hline & & Descarta a campo & 68,9 & 55,1 & 0,011 & 63,5 & 25,0 & 0,012 \\
\hline & & Basura domiciliaria & 37,0 & 14,5 & 0,009 & 26,0 & 0 & - \\
\hline Agujas & Clínica & Basura domiciliaria & 36,4 & 40,0 & 0,709 & 38,3 & 0 & - \\
\hline \multirow{2}{*}{ Cuchillo } & \multirow[t]{2}{*}{ Necropsia } & \multirow{2}{*}{$\begin{array}{l}\text { Usa en otras tareas } \\
\text { Esteriliza }\end{array}$} & 8,5 & 8,1 & 0,893 & 9,5 & 0 & - \\
\hline & & & 7,2 & 8,2 & 0,736 & 6,8 & 33,3 & 0,009 \\
\hline
\end{tabular}




\section{Bibliografía}

Baker WS, Gray GC. 2009. A review of published reports regarding zoonotic pathogen infection in veterinarians. J. Am. Vet. Med. Assoc. 234: 1271-1278.

de Titto E, Montecchia M, Brunstein L, Chesini F. 2015. Normativas para la gestión de residuos biopatogénicos. Rev. Arg. Salud Pública 6: 7-14.

Huertas PS, León AA, Tarabla HD. 2018. Zoonosis and veterinary waste disposal in rural practice. Rev. Arg. Microbiol. Aceptado, en prensa. doi.org/10.1016/j.ram.2018.08.004

Imoberdorf CG, Signorini ML, Tarabla HD. 2017. Adopción de prácticas seguras por veterinarios clínicos. XXXVI Jorn. CMV Provincia de Córdoba, Villa Giardino, Córdoba, CD.

López Cepeda M, Andrade Becerra RJ, Tarabla HD, Signorini ML, Molineri Al. 2014. Factores asociados con la presentación de accidentes laborales en veterinarios zootecnistas de departamento de Boyacá. Rev. Salud Uninorte (Colombia) 30: 23-30.

McLean M, Watson HK, Muswema A. 2007. Veterinary waste disposal: practice and policy in Durban, South Africa (20012003). Waste Manag. 27: 902-911.

Molineri Al, Signorini ML, Pérez L, Tarabla HD. 2013. Zoonoses in rural veterinarians in the central area of Argentina. Aust. J. Rur. HIth. 21: 285-290.

Navarrete MB, Tarabla HD. 2018. Factores asociados a riesgos ocupacionales y uso de elementos de protección personal en la práctica veterinaria con bovinos y equinos. InVet 20: 255-266.

Signorini ML, Pérez L, Tarabla HD, Molineri Al. 2014. Accidentes laborales en veterinarios rurales. Avances Cien. Vet. (Chile) 29: 36-41.

Scott E, Bloomfield SF. 1990. The survival and transfer of microbial contamination via cloths, hands and utensils. J. Appl. Bacteriol. 68: 271-278.

Tarabla HD. 2017. Riesgos laborales en Medicina Veterinaria en América Latina y el Caribe. Rev. Cien. Vet. (Costa Rica) 35: 65-84.

Tarabla HD, Hernández Villamizar AC, Molineri Al, Signorini ML. 2017. Percepción y prevención de riesgos ocupacionales en veterinarios rurales. Rev. Vet. UNNE 28: 152-156.

Wright JG, Jung S, Holman RC, Marano NN, McQuiston JH. 2008. Infection control practices and zoonotic disease risks among veterinarians in the United States. J. Am. Vet. Med. Asoc. 232: 1863-1872. 